\title{
Monitoring the impact of COVID-19 pandemic on mental health: a public health challenge? Reflection on Italian data
}

\author{
Maria Rosaria Gualano ${ }^{1} \cdot$ Giuseppina Lo Moro ${ }^{1}$ Gianluca Voglino ${ }^{1}$ (1) $\cdot$ Fabrizio Bert $^{1,2} \cdot$ Roberta Siliquini $^{1,2}$
}

Received: 5 August 2020 / Accepted: 30 September 2020 / Published online: 9 October 2020

c) Springer-Verlag GmbH Germany, part of Springer Nature 2020

\begin{abstract}
A recent Italian report on medicines use during COVID-19 epidemic outlined a non-significant increase in outpatient pharmaceutical antidepressant consumption in March and a significant increase in anxiolytic consumption. Along with this, an analysis of psychiatric hospitalizations in Lombardy revealed a reduction in voluntary admissions in the 40 days after the beginning of COVID-19 epidemic in Italy. Nevertheless, several studies reported a greater prevalence of depressive and anxiety symptoms in the Italian general population during the lockdown compared to before the pandemic. Furthermore, the request for psychological support by the Italian population appeared to be high during lockdown. Indeed, the Italian Ministry of Health declared that more than 50,000 calls to the telephone number for psychological support activated by the Ministry of Health and the Civil Protection because of the pandemic, with peaks during the lockdown. In addition, looking at Google Trends, the greatest searching activity for "psychological support" in recent years was detected the week of the 26th April 2020, followed by the week of the 22nd March 2020. We think that stronger indicators of mental health status and psychological well-being should be found to understand the long-term effects of the pandemic. The necessity of research for population-level and universal strategies is urgent, through repurposing, developing, and testing interventions to create evidence-based action plans for the entire population. Lastly, it is also essential to keep offering a psychological support suitable for all as done in past months to help individuals who have fewer opportunities to access care.
\end{abstract}

At the end of July, the Italian Medicines Utilisation Monitoring Centre (Osservatorio Nazionale sull'impiego dei Medicinali) released the report on medicines use during COVID-19 epidemic [1]. Considering outpatient pharmaceutical consumption of antidepressants before (January and February 2020) and after (March and April 2020) lockdown, no significant difference was reported in the packs $\times 10,000$ inhabitants/die ( $p=0.957)$ [1]. In March, an increase was noted (not statistically significant), probably due to a greater provision with the lockdown approaching [1]. On the other hand, in the 3-month period after lockdown, anxiolytic drugs privately purchased by citizens showed a significant slight increase $(p<0.001)$ [1]. Also in this case, a higher purchase was recorded in March when the lockdown began [1]. In addition, Clerici et al. conducted an analysis of psychiatric

Gianluca Voglino

gianluca.voglino@unito.it

1 Department of Public Health Sciences and Paediatrics, University of Torino, Via Santena 5 bis 10126, Torino, Italy

2 AOU City of Health and Science of Turin, Torino, Italy hospitalizations in Lombardy, the Italian region most affected by the pandemic, by considering the 40 days after the beginning of COVID-19 epidemic in Italy, compared to both the months before pandemic and the year before [2]. The findings revealed a reduction in voluntary admissions. Specifically, admissions for mood disorders showed a significant decrease, while no significant reduction was reported for the category defined as "other diagnoses", which included anxiety disorders [2]. Among the possible reasons for these observations, the authors suggested the fear of contamination and the avoidance of hospitals, a change in thresholds for hospitalization, and an increase in outpatient activities. A reduction in morbidity rates was considered to be less likely instead [2].

However, because of the wide spectrum of symptoms that mental health conditions might have, the consequences of the pandemic on mental health of general population in the short term are estimated with difficulty through indicators such as medications like antidepressants and hospital admission rates. Indeed, a broad range of severity of symptoms can exist, especially focusing on depressive and anxiety disorders. Although the above-mentioned indicators 
did not show an important increase during initial phases of pandemic, several studies reported a greater prevalence of depressive and anxiety symptoms in the Italian general population during the lockdown compared to before the pandemic [3-5]. For instance, the percentage of participants with depressive symptoms was reported to be $24.7 \%$ [3], $21.2 \%$ [4], and $32.4 \%$ [5]. Such percentages seem very high considering that the latest data before the pandemic, published by the Italian National Statistical Institute, estimated a prevalence of depressive symptoms of $5.4 \%$ in the Italian general population [6].

Furthermore, the request for psychological support from the Italian population appeared to be high during lockdown. Indeed, the Italian Ministry of Health declared more than 50,000 calls to the telephone number for psychological support activated by the Ministry of Health and the Civil Protection because of the pandemic, with peaks during the lockdown [7]. Interestingly, looking at the searches for "psychological support" in Italy in Google Trends, the greatest searching activity in recent years was detected the week of the 26th April 2020, followed by the week of the 22nd March 2020 [8] (Fig. 1). These dates correspond to the week before the end of the lockdown (3rd May [9]) and the week after the beginning of the lockdown (9th March [10]), thus suggesting that further investigations may be required to better understand the impact of lockdown measures at population level.

Given the above, we think that stronger indicators of mental health conditions and mental well-being of the general population should be found to understand the long-term effects of the pandemic and consequent measures on the entire population, in addition to the effects on high-risk groups. In these months, the monitoring and the comprehension of the impact on mental health in all its shades of severity might be useful to plan and implement strategies to promote resilience. Although the abovementioned works [3-5] were relevant because they contributed to increase knowledge about mental health of general population and explore potential risk and protective factors, they had some limitations (e.g., recruitment through social media, opportunity sampling) that need to be solved to realize an appropriate surveillance during this period. Therefore, we identified some key points that must be taken into account for monitoring the impact of the pandemic on mental health of general population.

First of all, the enrolment of participants cannot be exclusively online through social media. This approach limits the population that can access the survey. Similarly, the use of convenience sampling should be avoided. A joint action involving professionals working in the public health, mental health and primary care fields is required. In our opinion, General Practitioners represent a key players in the comprehensive care of individuals and might help not only to reach a more representative sample but also to meet unexpressed needs.

Additionally, the selection of the outcomes that need to be studied is a crucial point to be addressed. Although investigating psychological distress and symptoms of the most common mental disorders such as depression and anxiety is essential, a comprehensive evaluation of mental health must consider also mental well-being. Mental well-being is not just the flipside of psychological distress nor the absence of mental illness, but it is an important indicator of overall mental health status [11].

Therefore, we believe that the tools used for these evaluations must consider at least the following features: the tool has been validated for the screening in general population and not only tested in high-risk groups or in individuals with a diagnosis; the tool has been validated for the ages that represent the target of the survey; the tool has been validated
Fig. 1 "Psychological support" search in Italy (Google Trends data)

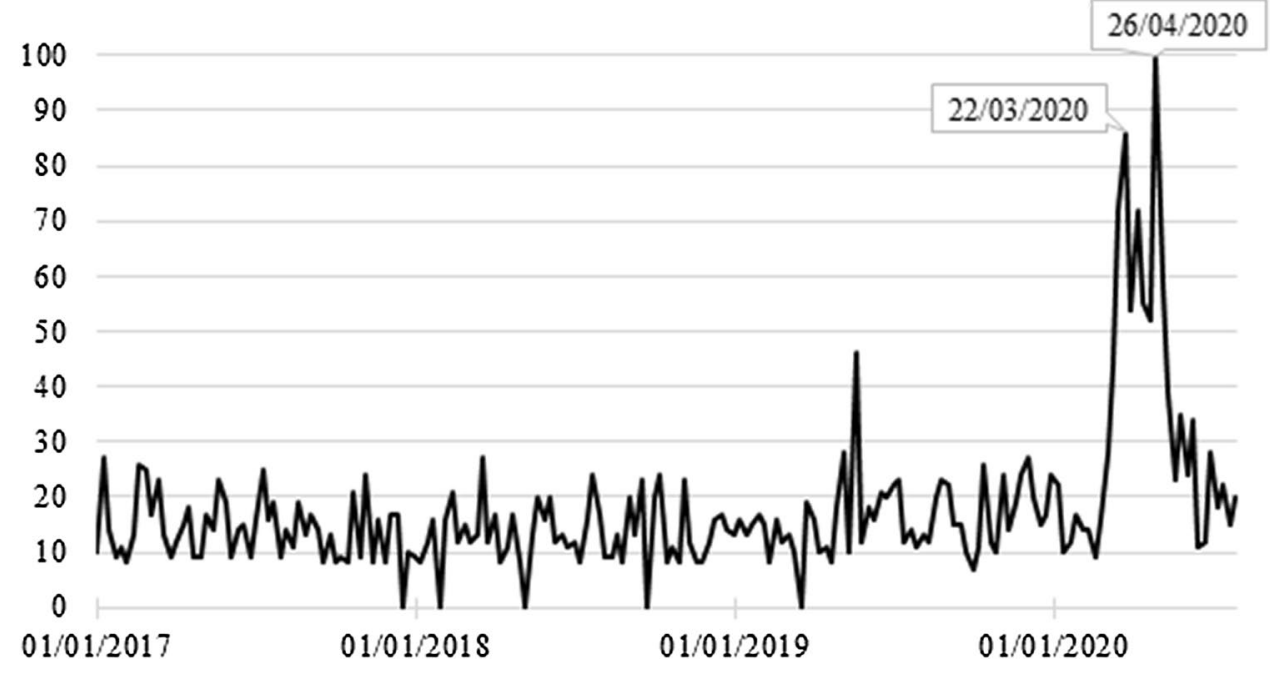


across different ethnicities and cultures. Furthermore, the measurement of the prevalence of mental health outcomes should be associated with an in-depth investigation on potentially vulnerable subgroups of general population and factors that can influence the impact on mental health. We think that, in addition to the study of risk factors, it would be advisable to realize a project that includes positive aspects, to understand all determinants that could possibly condition the effects of the pandemic and that could be used in the development of targeted intervention. Indeed, the pandemic brought out the relevance of planning long-term strategies to protect and promote good mental health, including interventions based on positive components, understanding positive social resources and boosting resilience [12]. Lastly, national or international studies should be implemented to avoid a high heterogeneity and fragmentation of methods and results. In this context, the necessity of research for population-level and universal strategies is urgent, through repurposing, developing and testing interventions to create evidence-based action plans [12].

\section{Compliance with ethical standards}

Conflict of interest On behalf of all authors, the corresponding author states that there is no conflict of interest.

\section{References}

1. Osservatorio Nazionale sull'impiego dei Medicinali (2020) Rapporto sull'uso dei farmaci durante l'epidemia COVID-19 Anno 2020. Agenzia Italiana del Farmaco, Roma

2. Clerici M, Durbano F, Spinogatti F, Vita A, de Girolamo G, Micciolo R (2020) Psychiatric hospitalization rates in Italy before and during COVID-19: did they change? An analysis of register data. Ir J Psychol Med. https://doi.org/10.1017/ipm.2020.29

3. Gualano MR, Lo Moro G, Voglino G, Bert F, Siliquini R (2020) Effects of Covid-19 lockdown on mental health and sleep disturbances in Italy. Int J Environ Res Public Health 17(13):4779. https ://doi.org/10.3390/ijerph17134779

4. Cellini N, Canale N, Mioni G, Costa S (2020) Changes in sleep pattern, sense of time and digital media use during COVID-19 lockdown in Italy [published online ahead of print, 2020 May 15]. J Sleep Res. https://doi.org/10.1111/jsr.13074

5. Mazza C, Ricci E, Biondi S et al (2020) A Nationwide Survey of psychological distress among Italian people during the COVID19 Pandemic: immediate psychological responses and associated factors. Int J Environ Res Public Health 17(9):3165. https://doi. org/10.3390/ijerph17093165

6. ISTAT Istituto Nazionale di Statistica (2018) La salute mentale nelle varie fasi della vita. Anni 2015-2017. https://www.istat.it/it/ files//2018/07/Report_Salute_mentale.pdf Accessed 5 Aug 2020

7. Italian Ministry of Health (2020) Press release n.189, 11 June 2020. Oltre 50mila telefonate al numero verde 800.833.833. Supporto psicologico attivo per tutto il mese di giugno. https://www. salute.gov.it/portale/nuovocoronavirus/dettaglioComunicatiN uovoCoronavirus.jsp?lingua $=$ italiano\&id $=5570$ Accessed 5 Aug 2020

8. Google Trends: "support psicologico" in Italy, last 5 years. https://trends.google.it/trends/explore?date=today $\% 25205$ -y\&geo=IT\&q=supporto\%2520psicologico Accessed 5 Aug 2020

9. Government of Italy Decree of the president of the Council of Ministers 10 April 2020. https://www.gazzettaufficiale.it/eli/ id/2020/04/11/20A02179/sg Accessed 30 May 2020

10. Government of Italy Decree of the president of the Council of Ministers 9 March 2020. https://www.gazzettaufficiale.it/eli/ id/2020/03/09/20A01558/sg Accessed 30 May 2020

11. Ryff CD (2014) Psychological well-being revisited: advances in the science and practice of eudaimonia. Psychother Psychosom 83(1):10-28. https://doi.org/10.1159/000353263

12. Holmes EA, O'Connor RC, Perry VH et al (2020) Multidisciplinary research priorities for the COVID-19 pandemic: a call for action for mental health science. Lancet Psychiatry S2215-0366(20):30168-30171 